\title{
Analysis of the structure of random packings of powder particles in laser additive technologies
}

\author{
Oleg Kovalev"1,2,*, Andrey Gusarov², and Vitaly Belyaev ${ }^{1}$ \\ ${ }^{1}$ Khristianovich Institute of Theoretical and Applied Mechanics SB RAS, Institutskaya str., 4/1, Novosibirsk, 630090, Russia \\ ${ }^{2}$ Moscow State Technological university “STANKIN", Vadkovsky per., 3-A, Moscow, 127055, Russia
}

\begin{abstract}
Under consideration are the peculiarities of the computer simulation of random packings of the particles involved in the laser additive technologies of selective laser melting and sintering of powders. The results of definition of random packings of monodisperse solid spheres are presented. Numerical analysis and structural investigations of their morphology are carried out. The peculiar character of the theoretical and computer models used for the analysis of particle packings in real powder media is understood. The technique of ray tracing is proposed for the description of laser radiation absorption and scattering in the layer of the stainless steel powder with the structure of the loose packing of mono-size spherical particles.
\end{abstract}

\section{Methods of random packings generation in a granular medium}

The physics of laser beam and powder layer interaction includes such processes as radiation absorption and scattering, heat transfer, powder consolidation and formation of a remelted trace. These processes are important for the understanding of the additive laser technologies of selective laser melting (SLM) and selective laser sintering (SLS) of powders [1]. The properties of the fabricated parts depend on the internal structure of the particle layer, and also on the above mentioned processes. The problem of formation of dense powder packings via varying the shape and the size of the particles is today a complex problem focusing much attention [2 - 4]. The dependencies of the packing structure on the particle size, the density, the specific surface and the pore connectivity degree are the important characteristics.

Computer simulation is a helpful tool to study the structural peculiarities of the random packing, which can be used for the description of the laser interaction processes on the micro- and macro-scale levels.

The methods of generation of random packings of the granular material can be divided into the dynamic methods of expansion or compression of a randomly chosen free configuration of the particle system, and the methods of consequent filling by particles of a certain space in the force field as the packing is formed in a restricted region. Each method is normally adopted to the conditions of packing usage for the solution of a specific task [2 - 3]. In the case of the selective laser melting or sintering, the powder is deposited on a substrate in layers with a scraper blade or a roller, and any possibility of compaction is excluded. It is believed that the density and the structure of the powder layer deposited before the laser action approaches to the density and the structure of the loose random packing [1].

\subsection{Method of generation of the random packing of spheres}

This paper uses the previously developed technique of calculation of the packings of monodisperse spherical particles [5]. The algorithm of calculation of the particle center coordinates in the loose layer is based on the simulating consequence of individual sphere throwing. At the inelastic collision, their adhesion interaction is taken into account, which permits having the loose packings with different porosity via the variation of the physical properties and the size of the particles.

The mathematical problem is formulated in the terms of the discrete elements method. A cuboid-shaped container with the sizes of $L_{x} \times L_{y} \times L_{z}$ is considered in the Cartesian system of coordinates $O x y z$. The rib sizes are preset as follows: $L_{x}=L_{y}=M D$, where $M$ is the number of sphere diameters $(10,15,20)$ arranged on the rib length. The container is filled via the consequent throw of $N$ individual solid spheres of the same diameter $D=2 R$. Initial coordinates of sphere centers $x_{0}$, $y_{0}, z_{0}$ are specified within the $\mathrm{z}_{0}=\mathrm{L}_{\mathrm{z}}$ distance from the substrate, and $x_{0}, y_{0}$ are chosen with the aid of the random number generator inside the preset range by the axes $O x, O y: 0<x_{0}<L_{x}, 0<y_{0}<L_{y}$. The container side faces and the bottom restrict the motion of the thrown spheres.

The sphere motion is caused by the gravity force with due regard to the adhesion interaction between spheres which is described by the Van-der-Waals forces. The interaction at the sphere collision is deemed to be

\footnotetext{
Corresponding author: kovalev@itam.nsc.ru
} 
absolutely non-elastic. In the collision instant, the speed of the incident sphere is assumed to be zero, its farther motion continues along the lying sphere surface in the pure sliding mode, and the rolling sphere may separate and lose the contact, hence continuing its free fall. As two or more contacts appear, providing that the total force remains positive, the thrown sphere stops, and the coordinates of its center are registered. The appearing adhesion force $F$ between the contacting solid spheres is directed along the line connecting their centers and results from inter-molecular interaction (from the Vander-Walls forces) [6]. For two similar spheres with the radius $R$ and distance between their surfaces $d$, the adhesion force $F$ is written as [6]:

$$
F(q)=-\frac{H_{a}}{12 R} \frac{(1+q)}{\left(q^{2}+q+q\right)^{2}\left(q^{2}+q+q+1\right)^{2}},
$$

For the particles contacting with walls:

$$
F(q)=-\frac{H_{a}}{48 R} \frac{1}{q^{2}(1+q)^{2}},
$$

where $q=d / R, H_{a}$ is the Hamaker constant. In the case of stainless steel, $H_{a}=2.1 \times 10^{-20} \mathrm{~J}$.

The details of the above described algorithm are presented in [5].

\subsection{Calculation of container filling}

Fig. 1 shows the $O x z$ projections of the filled containers for $D=0.5$ and $5.0 \mu \mathrm{m}$. As the sphere diameter $D$ decreases, the effect of the adhesion force on the packing structure becomes essential, container filling becomes more complicated, i.e. some opens appear between the walls and spheres, Fig. 1(a, b). In the presence of the adhesion and the gravity forces, the falling particles form the packing with an anisotropic structure.

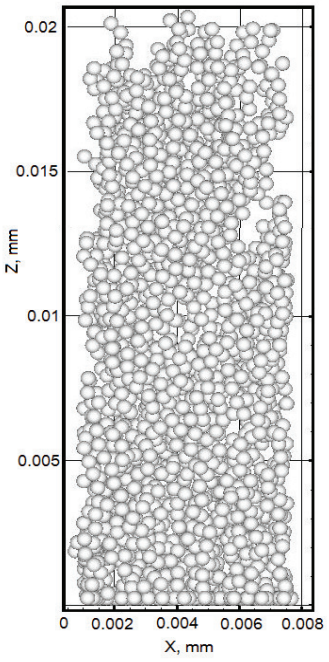

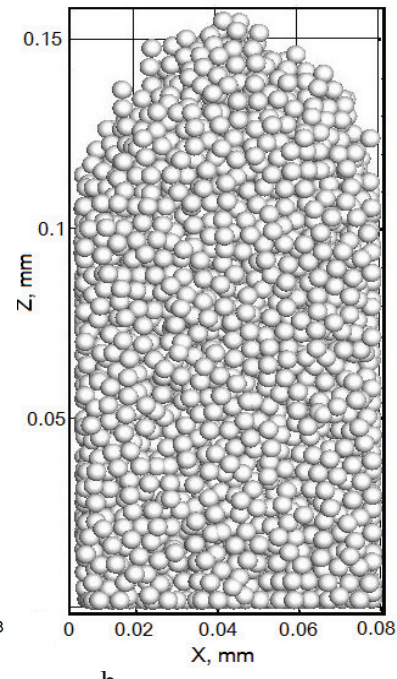

Fig. 1. Projections of the containers in the $O x z$ plane, sphere diameters $D, \mu \mathrm{m}: 0.5(\mathrm{a}) ; 5.0(\mathrm{~b})$
Owing to the adhesion forces which become more noticeable for the particles below $10 \mu \mathrm{m}$, the anisotropy appears to a greater extend over the container height.

\section{Structural parameters of the packing}

\subsection{Theory}

Such values as the porosity (or the density), the particle centers distribution, the pore specific surface and the tortuosity are the structural parameters of the packings which can be measured experimentally in packed granular media.

\subsubsection{Porosity}

Let the sphere of the radius $R$ and center at the height $z_{i}$ (Fig. 2(a)) cut out a circle of the area $\pi\left(R^{2}-\left(z_{i}-z\right)^{2}\right)$ on the horizontal plane $z$.

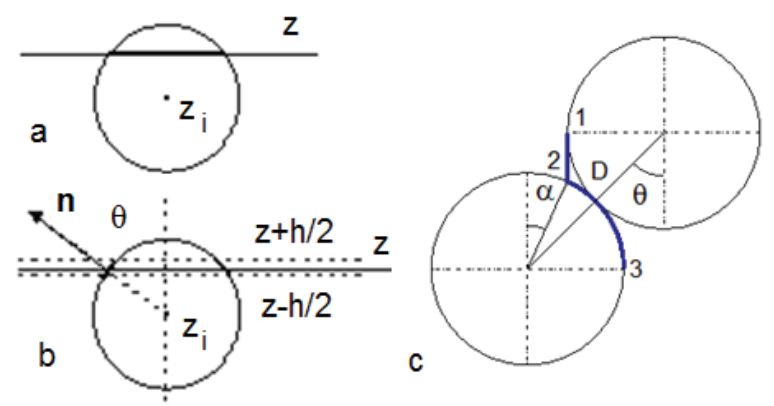

Fig. 2. Calculation of the porosity (a), the specific surface (b) and the tortuosity (c) for two adjoining spheres of the packed layer

If the area of the cross of the plane $z$ and the container equals to $S(z)=$ const, the value of the surface porosity $\varepsilon_{S}$ or cross-section porosity at the $z$ level equals to the fraction of this area free from spheres:

$$
\varepsilon_{S}(z)=1-\frac{\pi}{S} \sum_{i} \max \left[0, R^{2}-\left(z_{i}-z\right)^{2}\right]
$$

To calculate the volume porosity $\varepsilon_{V}$, let us consider the volume $V(z)=S z$ at the height $z$. Taking into account that the bottom area also equals to $S$, the volume porosity $\varepsilon_{V}$ is:

$$
\varepsilon_{V}(z)=1-\frac{4 \pi R^{3}}{3 S} \frac{1}{z} \sum_{i} \chi\left(z-z_{i}\right),
$$

where $\chi(x)$ is the Heaviside unit function, and the summing includes every sphere in the container.

Comparison of two ways (2a) and (2b) of calculating the packing porosity is of interest. According to [7], in the case of the homogeneous granular medium, the porosity value approximately equals to the value of the volume porosity of the system 


$$
\widehat{\varepsilon}_{S} \approx \varepsilon_{V} .
$$

It is necessary to determine the validity range for this equation in our conditions.

\subsubsection{Height distribution of sphere centers}

The sphere area cut out by the layer of the thickness $h$, centered about the plane $z$, Fig. 2(b), is $2 \pi R h$, whereas the angle $\theta$ between the external normal $\mathbf{n}$ and vertical axis $z$ is found in the equation: $\cos \theta=\left(z-z_{i}\right) / R$. Amount of sphere centers at the height $z$ is calculated in accord with (3)

$$
N_{C}(z)=\sum_{i} \frac{\max \left[0,(h / 2)^{2}-\left(z_{i}-z\right)^{2}\right]}{(h / 2)^{2}-\left(z_{i}-z\right)^{2}},
$$

where $h$ is the height-wise subinterval. The formula (3) permits calculating the amount of the particles, which centers coordinates $z_{i}$ get at the height $z$ in the band $z_{i} \in(z-0.5 h, z+0.5 h)$.

\subsubsection{The specific surface}

The specific surface at the $z$ level is found as the ratio of the sums of sphere surface areas inside this layer to its volume $S h$ :

$$
A(z)=\frac{2 \pi R}{S} \sum_{i} \frac{\max \left[0, R^{2}-\left(z_{i}-z\right)^{2}\right]}{R^{2}-\left(z_{i}-z\right)^{2}}
$$

The sum in the right part (4) equals in fact to the amount of the particles which centers lie inside the layer restricted by the planes $z-R$ and $z+R$.

Let the spatial distribution of the spheres be specified by the probability of sphere center finding in the volume unit $n(z)$. Then the sums in the equations (2a) and (4) can be approximately alternated by the integrals:

$$
\begin{gathered}
\varepsilon=1-\pi \int_{z-R}^{z+R}\left[R^{2}-\left(z^{\prime}-z\right)^{2}\right] n\left(z^{\prime}\right) \mathrm{d} z^{\prime} \\
A=2 \pi R \int_{z-R}^{z+R} n\left(z^{\prime}\right) \mathrm{d} z^{\prime}
\end{gathered}
$$

If $n$ does not depend on $z$, i.e. the packed layer is statistically homogeneous, integration gives the followings results: $\varepsilon=1-4 / 3 \pi n R^{3}, A=4 \pi n R^{2}$. Hence, $n$ can be excluded:

$$
A=\frac{3(1-\varepsilon)}{R}=\frac{6(1-\varepsilon)}{D}
$$

As a result, we have the connection (7) between the porosity $\varepsilon$, specific surface, and mono-diameter $D$ of the particle packing.

\subsubsection{The pore tortuosity}

The pore tortuosity characterizes the percolation properties of the packed structures and is found as the ratio of the path length of the tracing point around the porous structure labyrinth to the value of the shortest distance between the initial and final position of this point. Let us consider the passage of such a point though the packed layer pores by the shortest way under the action of the constant external force. Let the force be directed vertically downward. The path part 1-2-3 between two adjoining spheres with the diameter $D$ is shown in Fig. 2(c). The geometry is specified by the inclination angle $\theta$ of the line connecting the sphere centers. The angle $\alpha$ is found in the equation: $\sin \alpha=2 \sin \theta-1$. It follows from Fig. 2(c) that the height difference between the points 1 and 3 is $H=D \cos \theta$, whereas the path $1-2-3$ is $S=H+0.5 D(0.5 \pi-|\alpha|-\cos \alpha)$. The path 1-2-3 tortuosity for this angle $\theta$ is determined by the relation $S / H=1+\Omega-\Psi$, where $\Omega=(0.5 \pi-|\alpha|) /(2 \cos \theta)$, $\Psi=(\cos \alpha) /(2 \cos \theta)$.

Then the packed layer tortuosity is found as:

$$
\tau=1+\langle\Omega\rangle-\langle\Psi\rangle
$$

where $\langle\ldots\rangle$ is the averaging operator, which si defined as the averaging by the angle $\theta$ in the $2 \mathrm{D}$ case (2D) and by the solid angle in the 3D case (3D).

\subsection{Calculation of the packing parameters}

Fig. $3(\mathrm{a}, \mathrm{b})$ presents the varying surface $\varepsilon_{S}$ and volume $\varepsilon_{V}$ porosity over the container height $z$. Fluctuations $\varepsilon_{S}$ and $\varepsilon_{V}$ are most noticeable at low $z$, which are caused by the effect of the container lower boundary (bottom).

Due to the stochastic distribution of the sphere centers $N_{C}$, Fig. 4(a), the medium becomes more uniform at $z>0.1$, and a certain fluctuation amplitude and frequency $\varepsilon_{S}$ settles, Fig. 3(a). The monotonous variation of $\varepsilon_{V}(z)$, Fig. 3(b), is related with the fact that, according to (2b), the mean-volume porosity value is calculated in fact for each $z$. Based upon Fig. 3(a) and $3(b)$, the approximate equation (2c) is satisfied; this equation is valid for the homogeneously ordered granular structures, and is theoretically vindicated in the theory of granular and saturated porous media [7]. It is interesting to check the satisfiability of the ratio (2c) for the case of polydisperse packings.

Indeed, the distribution of the center coordinates $N_{C}(z)$, Fig. 4(a), in each container is uniform on average, except for the case $D=0.5$ and $1.0 \mu \mathrm{m}$. At $z<3 h$, there are evident deviations. The reason is the container bottom effect, where smaller particles are concentrated denser. Fig. 4(b) presents the variation of the specific pore surface $A(z)$ over the container height. It is shown 
that for small particles with $D=0.5$ and $1.0 \mu \mathrm{m}$, the packed layer has quite a developed specific pore surface.
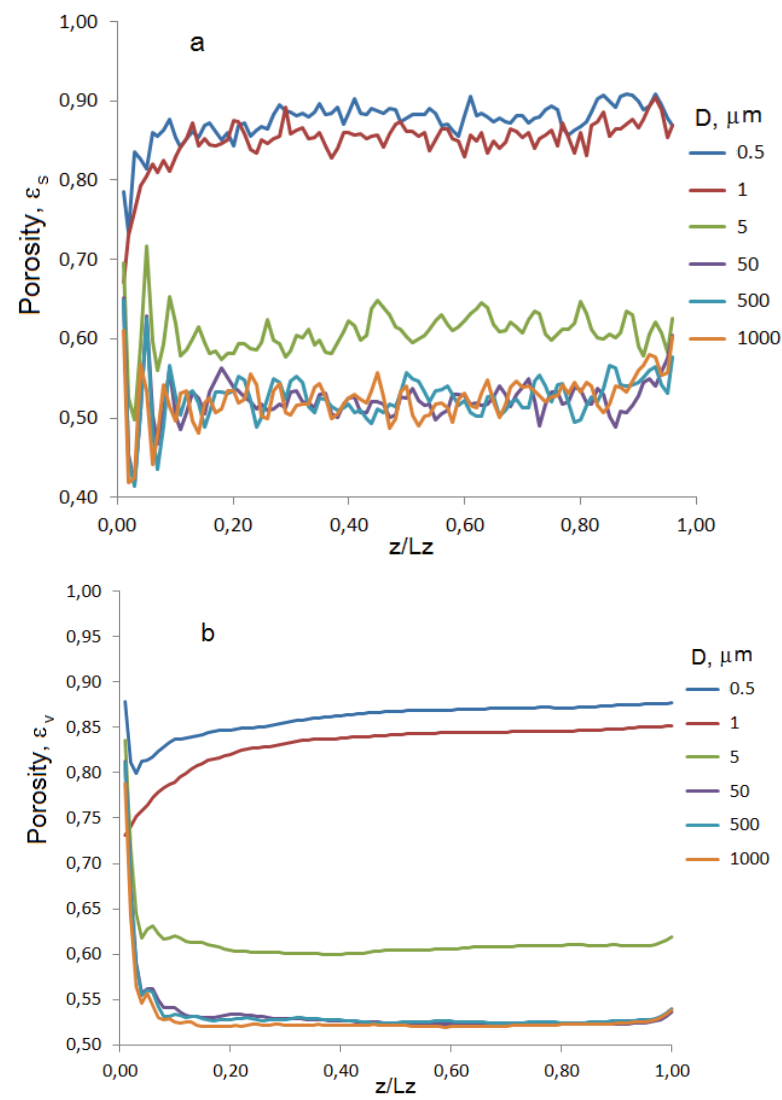

Fig. 3. Variation of the surface $\varepsilon_{S}$ (a) and volume $\varepsilon_{V}$ (b) porosity over the container height at the varying sphere monodiameter $D, \mu \mathrm{m}: 0.5 ; 1.0 ; 5 ; 50 ; 500 ; 1000$.

Fig. 5 demonstrates the 3D pore tortuosity over the height $z(D=5 \mu \mathrm{m})$, where the trajectories of nine tracing points around the pore labyrinth are presented. Vertical sections of the trajectories are shown by solid lines, enveloping sections of sphere surfaces are shown with markers.

Table 1 presents the numerical values of the structural parameters of the studied packings.

Table 1. Packing structure characteristics

\begin{tabular}{|c|c|c|c|c|c|}
\hline $\begin{array}{c}D, \\
\mu \mathrm{m}\end{array}$ & $\varepsilon_{V}$ & $\begin{array}{c}A, \mu \mathrm{m}^{-1} \\
\text { (theory) }\end{array}$ & $\begin{array}{c}\hat{A}, \mu \mathrm{m}^{-1} \\
\text { (calcula- } \\
\text { tion) }\end{array}$ & $\widehat{\tau}_{x}$ & $\widehat{\tau}_{z}$ \\
\hline 0.5 & 0.86 & 1.68 & 1.5 & 1.013 & 1.014 \\
\hline 1 & 0.84 & 0.96 & 1.0 & 1.015 & 1.014 \\
\hline 5 & 0.54 & 0.54 & 0.57 & 1.042 & 1.037 \\
\hline 50 & 0.47 & 0.05 & 0.058 & 1.048 & 1.053 \\
\hline 500 & 0.49 & 0.005 & 0.007 & 1.049 & 1.052 \\
\hline
\end{tabular}

According to the formula (7) obtained for the case of the homogeneous distribution of solid spheres, the theoretical values of the specific surfaces $A$ of monosphere packings, $D$ from 0.5 to $500 \mu \mathrm{m}$ were calculated by the values of the volume porosity $\varepsilon_{V}$ and monodiameter $D$.
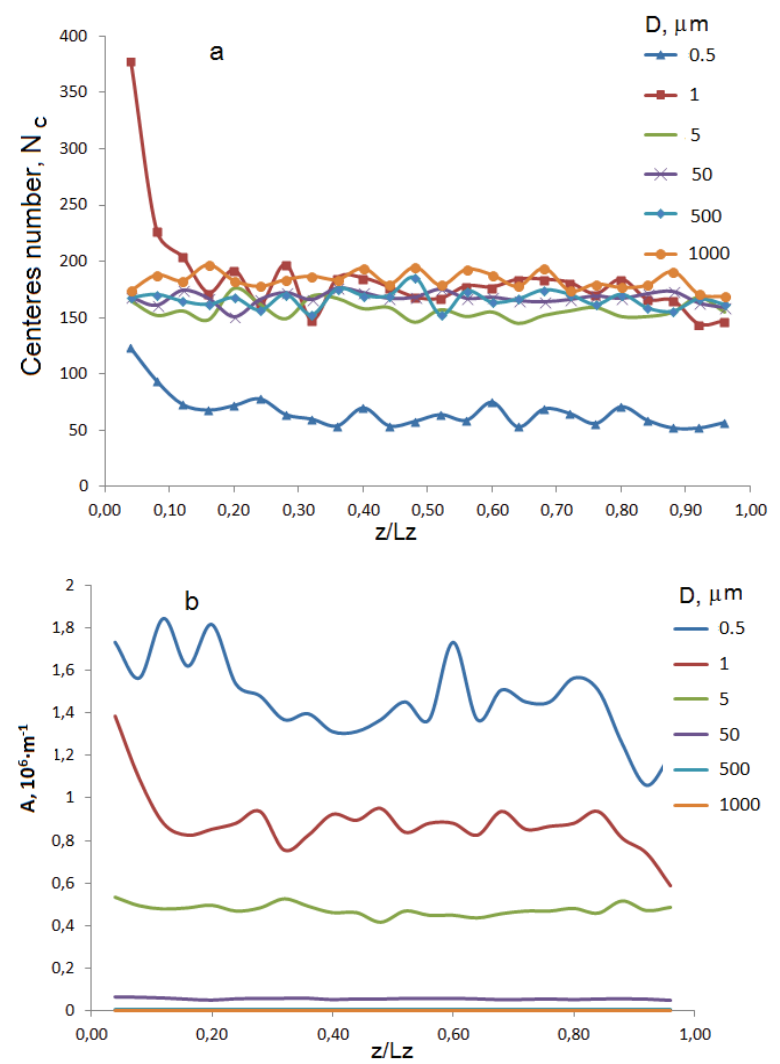

Fig. 4. Distributions of sphere center coordinates $N_{C}$ (a) and specific pore surface $A(b)$ over the container height at the varying mono-size of packing particles $D, \mu \mathrm{m}: 0.5 ; 1.0 ; 5 ; 50$; $500 ; 1000$.

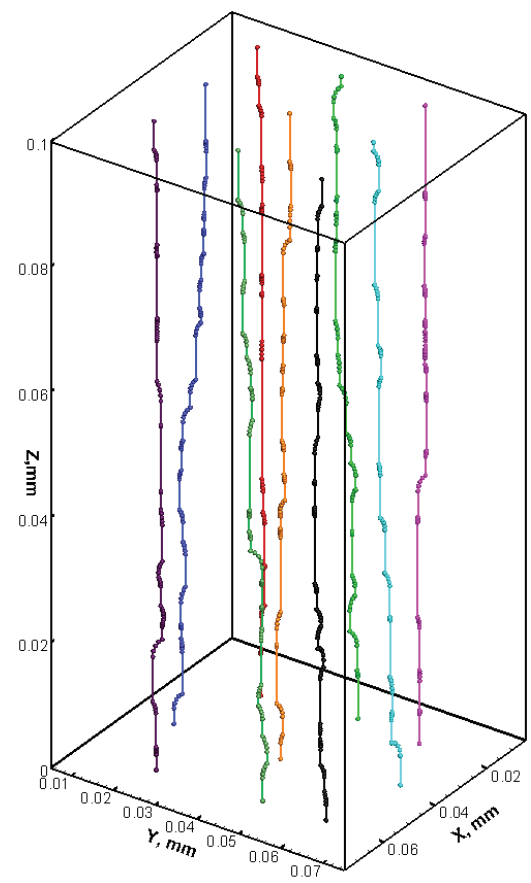

Fig. 5. Trajectories of the tracing points around porosity labyrinths calculated by the algorithm of Fig. 2(c) 
The average values of the specific surfaces $\hat{A}$, according to the calculation presented in Fig. 4(b), are in good agreement with the theoretical values of $A$, calculated by the formula (7), see Table 1 .

The characteristic (average) values of tortuosity $\hat{\tau}_{x}$, $\hat{\tau}_{z}$ of the studied packings in the directions $O x, O z$ are presented here, respectively.

Based upon the comparison of $\hat{\tau}_{x}, \hat{\tau}_{z}$, it is evident that, the structure anisotropy does not appear so highly for the mono-size packings, from the viewpoint of the percolation properties.

\section{Laser beam tracing method}

The physics of the laser and powder particle layer interaction is such that the absorption of the laser radiation by the particle surface goes along with repeated re-reflection and scattering over the layer depth. The above constructed mono-size packing of particles were used to define the algorithm of laser beam tracing based upon the Gaussian beam presentation in the form of the finite-number discrete luminous elements [8].

Initial cross section of the Gaussian beam $\pi \omega_{0}^{2}$ with the radius $\omega_{0}$ is divided into the finite number of discrete luminous elements $n_{x y}$ (LE): $\pi \omega_{0}^{2}=\sum_{k=1}^{n_{x y}} \Delta S_{k}$. Each LE has its initial position with the coordinates $x_{k}$, $y_{k}, z_{k}, \quad$ and initial radiation energy $\Delta E_{k}=I\left(x_{k}, y_{k}, z_{k}\right) \Delta S_{k}$. First, the LE move by the trajectories parallel to the $O z$ axis. When interacting with the particle surface, the LE trajectory changes its direction in accordance with the geometrical optics law (the incidence angle equals to the reflection angle). Its energy $\Delta E_{k}$ decreases by the value of the energy absorbed by the particle surface in the incidence point. The numerical algorithm includes summing of the absorbed energy for each spherical particle from each LE, including the multiply reflected ones. The energy summed in such a way is averaged over the sphere surface. The absorbed laser energy is then used in the thermal balance of individual particles [9].

Fig. 6 presents the pattern of LE trajectory positions in the granular layer of steel spherical particles of the same radius $(10 \mu \mathrm{m})$. The layer thickness is about $100-$ $120 \mu \mathrm{m}$. It is seen that the incident beam does not pass throughout the whole layer thickness. The upper part of the layer ( $2-3$ diameters) turns out to be heterogeneous at the construction due to the random particle throw.

The trajectories of multiply reflected LE penetrating into the layer depth are clearly seen. The temperature distribution over the particles in the layer is shown; it is calculated by the previously proposed model of discrete thermal elements [9]. Note that the thermal balance in this model regards only the absorbed energy of the laser radiation. Fig. 6 gives the calculation neglecting the contact thermal conductivity.

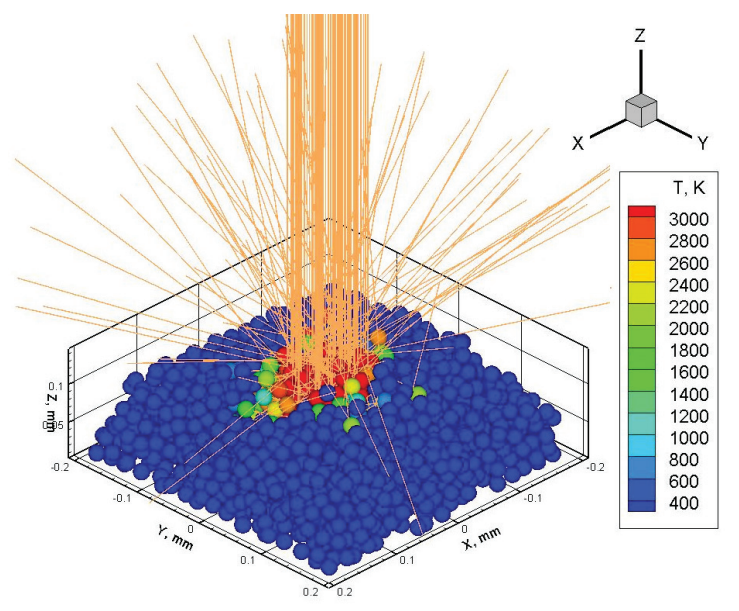

Fig. 6. Interaction of the laser beam and the randomly packed granular layer of spheres, 2,000 pieces. Particle material steel, radiation wavelength $1.07 \mu \mathrm{m}$, power $100 \mathrm{~W}$, beam radius $50 \mu \mathrm{m}$.

It is demonstrated that, to evaluate the laser radiation effect on powder materials, it is necessary to known the spatial distribution of the absorbed energy over the depth. The incident radiation may reflect repeatedly from the powder particle surface and penetrate inside. The proposed method consists of the detailed tracking of the LE path, with due regard to the interphase reflection and refraction.

\section{Conclusions}

The peculiarities of particle packing in powder systems have been considered. There are attractive Van-derWaals' forces between small particles. As the particles get closer, these forces appear to a higher degree. Under the gravity conditions, as the packing of spherical particles forms, these forces influence the contact interaction and the spatial distribution of the particles. This factor considerably influences the internal structure and the physical properties of the packing, i.e. the packing density, the pore specific surface and the tortuosity, which is very important for the SLM and SLS technologies. Computer simulation of metal powder packing has been performed. Specific character of utilization of the computer models is detected for the analysis of the particle packings in real powder media.

Simulation by the ray tracing method has demonstrated that the penetration of the laser radiation into the powder layer is especially important for highly absorbing and reflecting metal powders. Correct combination of fine and large particle fractions permits having the packing with the properties favorable for the penetration and absorption of the laser radiation. It allows to improve the quality of the produced parts, reach denser sintering of powders and achieve good performance properties.

The authors gratefully appreciate the financial support from the Russian Scientific Fund (contract No. 15-19-00254, May 18, 2015). 


\section{References}

1. I. Yadroitsev, Selective laser melting: Direct manufacturing of 3D_objects by selective laser melting of metal powders (Lambert Acad. Publ., 2010)

2. B.A. Klumov, Y. Jin, H.A. Makse, J. Phys. Chem. B. 118 (36), 10761-10766 (2014)

3. G. Parisi, F. Zamponi, Rev. Mod. Phys. 82 789-845 (2011)

4. S. Torquato, F.H. Stillinger, Rev. Mod. Phys. 82, 2633-2672 (2010)

5. O.B. Kovalev, I.O. Kovaleva, Journal of Applied Mechanics and Technical Physics 55, 709-717 (2014)

6. H.C. Hamaker, Physica IV 10, 1058-1072 (1937)

7. V.N. Nikolaevskij, K.S. Basniev, A.T. Gorbunov, G.A. Zotov, Mechanics of saturated porous media (Moscow: Nedra. 1970)

8. C.D. Boley, S.A. Khairallah, A.M. Rubenchik, Applied optics 54, 2477-2482 (2015)

9. O.B. Kovalev, A.V. Gusarov, Int. J. Thermal Sciences 114, 327-341 (2017) 